\title{
CSF3R, SETBP1 and CALR mutations in chronic neutrophilic leukemia
}

\author{
Yajuan Cui ${ }^{1}$, Bing Li', Robert Peter Gale², Qian Jiang ${ }^{3}$, Zefeng Xu' ${ }^{1}$, Tiejun Qin ${ }^{1}$, Peihong Zhang ${ }^{4}$, Yue Zhang ${ }^{1,5}$ \\ and Zhijian Xiao ${ }^{1,5^{*}}$
}

\begin{abstract}
The WHO 2008 definition of chronic neutrophilic leukemia (CNL) is based on clinical and laboratory parameters but not on molecular abnormalities. Mutations in CSF3R, SETBP1 and CALR are reported in patients with chronic neutrophilic leukemia (CNL). However, because CNL is rare, there are few large studies of this issue. We sequenced these genes in 14 patients who met the WHO-criteria of CNL. 8 subjects had CSF3R T6181 6 SETBP1 mutations and 1 a CALR mutation. Our data suggest mutation analysis of CSF3R, SETBP1 and CALR should be included in the diagnostic criteria for CNL. These data may also have therapy implications.
\end{abstract}

Keywords: Chronic neutrophilic leukemia, CNL, CSF3R, SETBP1, CALR, Mutation

\section{To the Editor}

The WHO defines chronic neutrophilic leukemia (CNL) as a myeloproliferative neoplasm (MPN) with sustained elevated neutrophils and $<10 \%$ immature cells [1]. Recently, recurrent somatic mutations in the membrane proximal domain of CSF3R were reported in patients with CNL [2,3]. CSF3R was mutated in 100\% [3], SETBP1 33\% [3] and CALR in $12.5 \%$ of WHO-defined cases of CNL [4]. We analyzed mutations in CSF3R, SETBP1 and CALR in 14 subjects who met the WHO-criteria.

\section{Findings}

CSF3R exon 14-17 [3], SETBP1 exon 4 [3] and CALR exon 9 [5] were amplified by PCR and sequenced. 8 subjects who met the WHO $2008 \mathrm{CNL}$ criteria had a

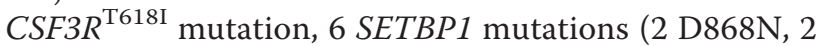
I871T, 1 G870S and 1 D874N) and the last had a CALR mutation (c.1154-1155insTTGTC). All mutations were heterozygous except 1 case of SETBP $1^{\mathrm{I} 871 \mathrm{~T}}$. 6 other subjects, 2 with monoclonal gammopathy of unknown significance (MGUS)-associated CNL and 4 with

\footnotetext{
* Correspondence: zjxiao@medmail.com.cn

1 MDS and MPN Centre, Institute of Hematology and Blood Diseases Hospital, Chinese Academy of Medical Sciences \& Peking Union Medical College, 288 Nanjing Road, Tianjin 300020, China

${ }^{5}$ State Key Laboratory of Experimental Hematology, Institute of Hematology and Blood Diseases Hospital, Chinese Academy of Medical Sciences \& Peking Union Medical College, Tianjin, China

Full list of author information is available at the end of the article
}

reactive neutrophilic leukocytosis had no mutation of these genes. No subject had a JAK2 ${ }^{\mathrm{V} 617 \mathrm{~F}}$ mutation (Table 1).

The consistent association between CSF3RT618I and CNL in our study is similar to data of Maxson et al. [2] and Pardanani et al. [3] (Table 2). Tefferi et al. [6] suggested including $C S F 3 R^{T 618 \mathrm{I}}$ or other membrane proximal CSF3R mutations as a criteria for diagnosis of CNL. We also confirmed the high incidence SETBP1 mutations in patients with CNL. The mutations we detected focused on a hotspot area from D868 to D874 (Table 1). Although these mutations also occur in other hematologic neoplasms such as atypical chronic myeloid leukemia aCML and chronic myelomonocytic leukemia (CMML), analysis of SETBP1 mutations could help distinguish CNL from reactive conditions such as infection, inflammatory conditions and non-haematologic neoplasms.

Gotlib et al. reported JAK2 ${ }^{\mathrm{V} 617 \mathrm{~F}}$ mutation in a subject of CNL [7]. Lasho et al. reported a CALR missense mutation in a subject with CNL [4]. We found concurrent $C S F 3 R^{\mathrm{T} 618 \mathrm{I}}$ and $C A L R$ frame-shift mutations in 1 subject. The 5 bp insertion into $C A L R$ exon 9 is reported in $B C R / A B L 1$ - and JAK2-negative MPNs and results in a $1+$ base-pair frame-shift with an altered C-terminus.

There is controversy whether co-existence of MGUS and CNL is one or two diseases. The 2 MGUS subjects in our study had no mutation in CSF3R, SETBP1, JAK2 or CALR. In another study, none of 6 cases of MGUS- 
Table 1 Clinical characteristics and laboratory variables

No. Diagnosis Gender/Age Hb (g/L) WBC $\left(\times 10^{9} / \mathrm{L}\right)$ ANC $\left(\times 10^{9} / \mathrm{L}\right)$ PLT $\left(\times 10^{9} / \mathrm{L}\right)$ Spleen $(\mathrm{cm}, \mathrm{LCM})$ Karyotypes CSF3R SETBP1 CALR Jurvival

\begin{tabular}{|c|c|c|c|c|c|c|c|c|c|c|c|c|c|c|}
\hline & & & & & & & & & & & & & & (month) \\
\hline 1 & $\mathrm{CNL}$ & M/80 & 120 & 27.19 & 25.42 & 91 & 3 & $\begin{array}{l}46, X Y, t(1,7) \\
(p 32, q 11)[10]\end{array}$ & T6181 & D874N & wt & wt & Hydroxyurea & 52 \\
\hline 2 & $\mathrm{CNL}$ & M/64 & 123 & 86.83 & ND & 394 & 0 & $46, X Y[5]$ & T6181 & D868N & wt & wt & Hydroxyurea & $5+$ \\
\hline 3 & $C N L$ & $\mathrm{~F} / 77$ & 125 & 35.68 & 25.92 & 351 & 4 & 46,XY[20] & T6181 & G870S & wt & wt & Hydroxyurea & $22+$ \\
\hline 4 & $\mathrm{CNL}$ & $F / 49$ & 104 & 85.61 & 79.47 & 20 & 6 & $46, X X[20]$ & T6181 & I871T & wt & wt & Hydroxyurea & $13+$ \\
\hline 5 & $\mathrm{CNL}$ & M/70 & 86 & 146.77 & 121.82 & 104 & 15 & ND & T6181 & I871T & wt & wt & Hydroxyurea & 17 \\
\hline 6 & $\mathrm{CNL}$ & $M / 43$ & 55 & 112.65 & 101.1 & 98 & 10 & $46, X Y[5]$ & T6181 & D868N & wt & wt & Hydroxyurea & $4+$ \\
\hline 7 & $\mathrm{CNL}$ & $F / 69$ & 102 & 57.40 & 40.16 & 231 & 6 & $46, X X[12]$ & T6181 & wt & $\begin{array}{l}\text { C.1154- } \\
\text { 1155insTTGTC }\end{array}$ & wt & Hydroxyurea & 10 \\
\hline 8 & $\mathrm{CNL}$ & M/45 & 119 & 32.76 & 28.73 & 286 & 6 & $46, X Y[9]$ & T6181 & wt & wt & wt & Hydroxyurea & 32 \\
\hline 9 & MGUS-CNL & M/46 & 63 & 65.30 & 60.7 & 101 & 7 & $46, X Y[2]$ & wt & wt & wt & wt & Hydroxyurea & $6+$ \\
\hline 10 & MGUS-CNL & $F / 52$ & 121 & 26.53 & 19.94 & 170 & 3 & $46, X X[20]$ & wt & wt & wt & wt & Hydroxyurea & $27+$ \\
\hline
\end{tabular}

All the parameters in Table 1 were measured at the initial diagnosis in our hospital.

MGUS-CNL: monoclonal gammopathy with uncertain significance associated CNL; Hb: hemoglobin; WBC: White Blood Cell Count; ANC: Absolute Neutrophil Count; PLT: Platelet Count; Spleen (cm): Spleen size under left costa. ND: not done. 
Table 2 CSF3R and SETBP1 mutations in CNL

\begin{tabular}{lllll}
\hline & $\begin{array}{l}\text { Gotlib J et al. } \\
\text { (2013) [7] }\end{array}$ & $\begin{array}{l}\text { Pardanani A } \\
\text { et al. (2013) [3] }\end{array}$ & $\begin{array}{l}\text { This } \\
\text { series }\end{array}$ & Total \\
\hline T618I only & 1 & 5 & 1 & 7 \\
T618I + SETBP1 & 4 & 4 & 6 & 14 \\
$\begin{array}{l}\text { Compound CSF3R } \\
\text { mutations }\end{array}$ & 2 & 1 & 0 & 3 \\
$\begin{array}{l}\text { Compound CSF3R } \\
\text { mutations + SETBP1 }\end{array}$ & 1 & 0 & 0 & 1 \\
$\begin{array}{l}\text { mutations } \\
\text { Others }\end{array}$ & $1^{\text {b }}$ & $2^{c}$ & & \\
\hline
\end{tabular}

a: compound CSF3R mutations mean nonsense or frameshift mutations that truncate the cytoplasmic tail (truncation mutations) combined with point mutations in the extracellular domain (membrane proximal mutation). In the 3 cases of compound CSF3R mutations Tyner et al. reported, two patients harbored T618I and one harbored T615A in the membrane proximal domain. In Tefferi's study, the compound CSF3R mutation

showed T6181 + c.2341 2342insC.

b: A case with JAK2 mutation only.

: A case with 1598 I and a case with M696T in CSF3R.

$d$ : A case with CSF3R T618I and CALR frameshift mutation.

associated CNL had CSF3R mutations [3]. Also, survival of patients with MGUS-associated CNL is significantly longer survival than those with CNL only. These data support the notion patients with MGUS and CNL are 2 diseases [8].

There may be therapy implications of our findings. CSF3R truncation mutations may be sensitive to SRC kinase-inhibitors such as dasatinib whereas CSF3R membrane proximal mutations may be sensitive to JAK kinase-inhibitors such as ruxolitinib $[9,10]$. Ruxolitinib was reportedly effective in a mouse model of CNL and a patient with CNL and a CSF3R ${ }^{\mathrm{T} 618 \mathrm{I}}$ mutation $[2,11]$. However, ruxolitinib was ineffective in a patient with CSF3R ${ }^{\mathrm{T} 618 \mathrm{I}}$ and SETBP1 mutations in whom fedratinib suppressed CFU-GM colony formation [12].

\section{Competing interests}

RPG acknowledges support from the NIHR Biomedical Research Centre funding scheme and is a part-time employee of Celgene Corp., Summit, NJ. The remaining authors declare no competing financial interests.

\section{Authors' contributions}

XZJ designed the study and drafted the article. CYJ collected the data, analyzed the molecular aberrations, and drafted the article. RPG drafted the typescript. LB, JQ, XZF, QTJ, ZPH, and ZY reviewed the clinical and pathology data. All authors read and approved the final typescript.

\footnotetext{
Acknowledgements

Supported in part by National Natural Science Funds (No.81370611, No. 81270585), Tianjin Key Natural Science Funds (12JCZDJC23900), National Public Health Grand Research Foundation (No.201202017) (to ZX). RPG acknowledges support from the NIHR Biomedical Research Centre funding scheme.
}

\section{Author details}

${ }^{1}$ MDS and MPN Centre, Institute of Hematology and Blood Diseases Hospital, Chinese Academy of Medical Sciences \& Peking Union Medical College, 288 Nanjing Road, Tianjin 300020, China. ${ }^{2}$ Hematology Research Centre, Division of Experimental Medicine, Department of Medicine, Imperial College, London, UK. ${ }^{3}$ Peking University People's Hospital, Peking University Institute of Hematology, Beijing, China. ${ }^{4}$ Department of Pathology, Institute of Hematology and Blood Diseases Hospital, Chinese Academy of Medical Sciences \& Peking Union Medical College, Tianjin, China. ${ }^{5}$ State Key Laboratory of Experimental Hematology, Institute of Hematology and Blood Diseases Hospital, Chinese Academy of Medical Sciences \& Peking Union Medical College, Tianjin, China.

Received: 10 September 2014 Accepted: 4 October 2014 Published online: 15 October 2014

\section{References}

1. Bain BJ, Brunning RD, Vardiman JW, Thiele J: Chronic neutrophilic leukemia. In WHO Classification of Tumors of Hematopoietic and Lymphoid Tissues. Edited by Swerdlow SH, Campo E, Lee Harris N. Lyon, France: IARC Press; 2008:38-39.

2. Maxson JE, Gotlib J, Pollyea DA, Fleischman AG, Agarwal A, Eide CA, Bottomly D, Wilmot B, McWeeney SK, Tognon CE, Pond JB, Collins RH, Goueli B, Oh ST, Deininger MW, Chang BH, Loriaux MM, Druker BJ, Tyner $\mathrm{JW}$ : Oncogenic csf3r mutations in chronic neutrophilic leukemia and atypical cml. N Engl J Med 2013, 368(19):1781-1790.

3. Pardanani A, Lasho TL, Laborde RR, Elliott M, Hanson CA, Knudson RA, Ketterling RP, Maxson JE, Tyner JW, Tefferi A: CSF3R T618I is a highly prevalent and specific mutation in chronic neutrophilic leukemia. Leukemia 2013, 27(9):1870-1873.

4. Lasho TL, Elliott MA, Pardanani A, Tefferi A: CALR mutation studies in chronic neutrophilic leukemia. Am J Hematol 2014, 89(4):450

5. Li B, Xu J, Wang J, Gale RP, Xu Z, Cui Y, Yang L, Xing R, Ai X, Qin T, Zhang Y, Zhang P, Xiao Z: Calreticulin mutations in chinese with primary myelofibrosis. Haematologica 2014, [Epub ahead of print].

6. Tefferi A, Thiele J, Vannucchi AM, Barbui T: An overview on CALR and CSF3R mutations and a proposal for revision of WHO diagnostic criteria for myeloproliferative neoplasms. Leukemia 2014, 28(7):1407-1413.

7. Gotlib J, Maxson JE, George TI, Tyner JW: The new genetics of chronic neutrophilic leukemia and atypical CML: implications for diagnosis and treatment. Blood 2013, 122(10):1707-1711.

8. Elliott MA, Dewald GW, Tefferi A, Hanson CA: Chronic neutrophilic leukemia (CNL): a clinical, pathologic and cytogenetic study. Leukemia 2001, 15(1):35-40.

9. Nicholson SE, Oates AC, Harpur AG, Ziemiecki A, Wilks AF, Layton JE: Tyrosine kinase JAK1 is associated with the granulocyte-colony-stimulating factor receptor and both become tyrosine-phosphorylated after receptor activation. Proc Natl Acad Sci U S A 1994, 91(8):2985-2988.

10. Corey SJ, Burkhardt AL, Bolen JB, Geahlen RL, Tkatch LS, Tweardy DJ: Granulocyte colony-stimulating factor receptor signaling involves the formation of a three-component complex with Lyn and Syk protein-tyrosine kinases. Proc Natl Acad Sci U S A 1994, 91(11):4683-4687.

11. Fleischman AG, Maxson JE, Luty SB, Agarwal A, Royer LR, Abel ML, MacManiman JD, Loriaux MM, Druker BJ, Tyner JW: The CSF3R T618I mutation causes a lethal neutrophilic neoplasia in mice that is responsive to therapeutic JAK inhibition. Blood 2013, 122(22):3628-3631.

12. Lasho TL, Mims A, Elliott MA, Finke C, Pardanani A, Tefferi A: Chronic neutrophilic leukemia with concurrent CSF3R and SETBP1 mutations: single colony clonality studies, in vitro sensitivity to JAK inhibitors and lack of treatment response to ruxolitinib. Leukemia 2014, 28(6):1363-1365.

doi:10.1186/s13045-014-0077-1

Cite this article as: Cui et al:: CSF3R, SETBP1 and CALR mutations in chronic neutrophilic leukemia. Journal of Hematology \& Oncology 2014 7:77. 